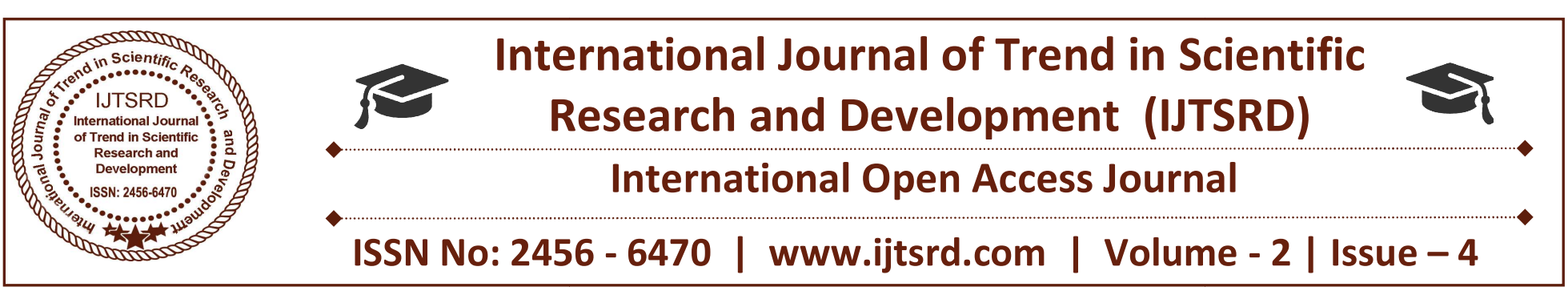

\title{
Image Retrieval for Minority Costume by using Color Histogram and Edge orientation (EO) Histogram Technique
}

\author{
S Shivakumar ${ }^{1}$, S Mallesh $^{2}$ \\ ${ }^{1}$ M.Tech, ${ }^{2}$ Assistant Professor \\ CMR Technical Campus, Medchal (M), Hyderabad, Telangana, India
}

\begin{abstract}
It is very significant to know the different minority costumes which are vanishing now a day which is used for minority culture protection and inheritance. To get the exact results for image retrieval there is need of a novel image feature representation method which is used to show the rich information of minority costume image. In this implementation first image is divided into image sub-blocks and then from that image calculate both color histogram as well as edge orientation histogram separately. The final feature used for minority costume image retrieval is fusion or integration of both color histogram and edge orientation histogram. At last improved Canberra distance is calculated for the input query image and retrieved output image. For the performance analysis there is use of self-build minority costume image dataset. MATLAB analysis results show that integrated technique of image retrieval will give outperforming results for characteristics such as color, texture, shape and spatial information, in comparison to existing technique proposed work will give higher and stable retrieval accuracy.
\end{abstract}

Keywords: Edge Orientation Histogram, Color Histogram, Minority Culture, Image Sub-blocks, Canberra Distance.

\section{INTRODUCTION}

The minority costume is the fundamental picture of the ethnic association distinguishing proof and the prized abundance of the Chinese nation. Where China is a country comprising of fifty six ethnic associations, and every one of them has its own one of a kind attire style with unmistakable ethnic qualities, because of the impact of different custom, conventions, and geological trademark. Be that as it may, with the quickening of overall financial and political mix in China, various minority outfit social conventions were quickly vanishing. This encouraged people to assume the survival of minority dress underneath the spic and span verifiable circumstance. Until further notice, the minority ensembles are uncommonly covered by method for historical centers statically. Contrasted and the ordinary insurance method of real firsts in exhibition halls, computerized wellbeing has longer assurance time and advances minority outfit lifestyle more noteworthy easily. Content-based thoroughly picture recovery is an exceptionally imperative topic inside the subject of test acknowledgment and manmade brainpower. It has been effectively connected to numerous fields, alongside medicinal conclusion, materials venture et cetera. The minority ensembles of same nation have their own extraordinary characters (bound together tone, style and examples.), which make them more prominent fabulous than ordinary home grown pix in picture preparing. In this manner, it is of top notch hugeness to examine the unmistakable abilities of minority outfits. In this venture, the virtual insurance of countrywide outfit is considered from the mentality of workstation vision.

In spite of the fact that nationwide minority apparel picture have confused visual capacities, the rule attributes by the by are attire shading, fabric surface and totem frame, which are as per the picture include in PC creative and perceptive. So we can utilize ordinary element extraction calculations to separate the capacities of minority dress pics. At blessing, an enormous number of strategies on extraction of shading, surface and shape capacities had been 
advanced and have just gotten reasonable impacts in heaps of fields.

Shading is the most prevailing and recognizing obvious trademark. The current shading highlight extraction techniques incorporate shade histogram [1], shading minute [2], shade lucidness vector [3] and shading correlogram [4]. In the present rendition of the MPEG-7 Final Committee Draft, various shading descriptors have been acknowledged comprehensive of wide assortment of histogram descriptors [5]. Surface is utilized to indicate the harshness or coarseness of thing surface and characterized as an example with some type of normality. Numerous scientists have suggest assorted calculations for surface assessment, which incorporate the outstanding dim degree co-event framework (GLCM) [6], closeby paired examples (LBP) [7], neighborhood directional styles (LDP) [8], et cetera. With the persistently growing of the utility field, new idea, similar to the hypothesis of wavelet, is brought. What's more, in 1996, Tai Sing Lee [9] utilized Gabor channels to separate surface abilities. Shape is the most basic capacity of the question. The ordinary shape descriptors are the second invariants [10], the Fourier rebuild coefficients [11] and the histogram of orientated slopes (HOG) [12].

The minority outfit photograph has exceptionally convoluted visual highlights, which make it more hard to be communicated through single element extraction set of tenets. So our motivation is to plan a trademark extraction set of standards in view of multi-highlights to express the measurements of minority photo completely. A ton of photo highlight extraction calculations in light of multi-abilities have been proposed as of late. In 2010, Guang-Hai Liu bears a solitary picture work outline approach, alluded to as multi-text on histogram [13], for photograph recovery. It organizes the advantages of co-occasion grid and histogram by strategy for addressing the property of co-occasion system the usage of histogram. Scaled down scale shape descriptor [14] proposed by technique for Guang-Hai Liu in 2011 is produced fundamentally based at the essential shades in littler scale structures with proportionate edge presentation. It viably incorporates shade, surface, and shape and shading format data as a whole for photograph recovery. Guang-Hai Liu also proposed shading qualification histogram [15] in 2013, which depend the perceptually uniform tinge refinement among factors underneath unmistakable foundations with respect to hues and angle introductions in $\mathrm{L}^{*} \mathrm{a} \mathrm{b}^{*}$ hue territory. The photograph trademark extraction calculations expressed above have all done exorbitant recovery precision inside the Corel photo database [16].

In context of various photograph work extraction computations in light of multi-limits have been basically associated in picture recuperation, this wander displays a sweeping feature descriptor to specific the rich perceptible capacities gave in minority dress picture. This descriptor is addressed with the guide of powerful blend of shade histogram and highlight presentation histogram. It's proposed in exploratory results that the photograph depiction frameworks used as a piece of our procedure are a convincing method for organizing low-level features into an aggregate.

Histogram has a wide range of sorts like histogram revolution, histogram dispersion, histogram exchanging, and histogram adjustment. Histogram evening out plays essential capacity in various virtual taking care of packs and also while the basic in a virtual photo is on the adjacent evaluation regards by using histogram alteration procedure we can manufacture the levels of world appraisal of various particular pix which has the fundamental data at the close-by appraisal esteems. By utilizing the histogram evening out forces of pixels related with computerized picture might be apportioned in higher approach to envision better through human unmistakable device. The critical pick up of the advanced picture histogram leveling is that it evens out the all estimations of pixels so the pixels with low forces can show signs of improvement visual appearance this is proficient by methods for spreading the better qualities to the low pixel esteems by means of the utilization of the histogram adjustment strategy.

\section{II.LITERATURE REVIEW}

CBIR has come protracted way sooner than 1990 and practically no activities have been posted by then, yet the scope of ventures posted in see that 1997 is developing. There are numerous CBIR calculations on the grounds that the after effect of these looks into and most extreme of these calculations strategy picture into a few layers of duties. Those layers of obligations join removing the multidimensional limits of a photo question and take a gander at it with pictures inside the database are perform after the machine populate database with photographs. Populating database with separated data from the previews and ordered as it ought to be affect the general execution of recovery. 
The actualities comprise of shade, shape, surface and whatever is left of picture work.

Highlights that most extreme approach centers around are shade, shape and surface. For shading, an across the board improvement over the RGB-tinge space utilization of adversary hue delineation makes utilization of the rival hue tomahawks (R-G, 2B-R-G, $\mathrm{R}+\mathrm{G}+\mathrm{B}$ ) is one approach to symbolize shade of a photograph.

There is additionally a route alluded to as Color Predominance Method which examines the photograph and replaces every pixel shading with the fresh out of the box new RGB tinge posting, gave an example ordering the utilization of surface in which a photograph is recorded by means of a vector (w1, w2, $\mathrm{w} 3, \mathrm{w} 4, \mathrm{w} 5, \mathrm{w} 6)$ speaking to the anticipated level of surface in which it is the extent of pixels grouped with surface they're presenting ordering the use of Intermediate Features.

However, a histogram with gigantic scope of compartments won't best blast the computational esteem, yet will likewise be in proper for developing green records for photograph measurements base. The customary shade histogram with quadratic shape (QF) separate as similitude degree and the thick hue histogram with Euclidean Distance about similar of their execution. In any case, they couldn't answer appropriately to moved or interpreted pix. In order to triumph over this issue invariant shading histogram framework is used makes which use of inclines in emerge channels that weight that weight the impact of a pixel at the histogram to balance the movements assisted by techniques for twists. At the point when a circumnavigated photo is given as the inquiry, the true picture is recovered in light of the fact that the nearest solid.

Color and Local Spatial Feature Histograms (CLSFH) has less trademark records and can catch more hue spatial data in a picture. At the equivalent time, as the four histograms utilized by CLSFH are computed all inclusive on the photograph, the two adjacent spatial measurement minute histograms and shading histogram are harsh to picture revolution, interpretation and scaling, the close-by directional qualification unit histogram is uncaring to picture interpretation and scaling. In CLSFH, the nonuniform quantized HSV hue display is used, a comparable old deviation of $5 \times 5$ neighbor of every pixel are determined, and are used to deliver the Local
Mean Histogram, the Local Standard Deviation Histogram; the Directional Difference Unit of three $\mathrm{X} 3$ neighbor of every pixel is described and handled, and is used to make the Local Directional Difference Unit Histogram. The 3 histograms and shading histogram are used as feature records to recoup shading picture. So CLSFH is serious for pictures, especially for portrayals with rather general surface and structure work.

Content Based Image Retrieval (CBIR) frameworks in light of shape the utilization of invariant picture minutes, similar to Moment Invariants (MI) and Zernike Moments (ZM) are to be had. MI and ZM are top at speaking to the frame elements of a photograph.

Be that as it may, non-orthogonality of MI and unpleasant remaking of $\mathrm{ZM}$ limit their product in CBIR. Quicker and remedy CBIR calculations are required for real time programs. This can be performed by methods for utilizing a classifier which incorporates Support Vector Machine (SVM), SVM is a regulated becoming more acquainted with approach utilized for picture compose. It perspectives the given photo database as two courses of action of vectors in a n dimensional space and fabricates a keeping isolated hyper flying machine that lifts the edge between the photos germane to address and the previews non important to the question. A CBIR machine the utilization of ELM capacities and ELM highlights with SVM as classifier.

\subsection{Histogram:}

A histogram is a graphical layout of the extent of pixels in a photo. In a more direct manner to give an illumination to, a histogram is a reference outline, whose $\mathrm{X}$-turn addresses the tonal scale, and $\mathrm{Y}$-center point addresses the measure of pixels in a photo in a particular area of the tonal scale. For example, the diagram of a luminance histogram exhibits the wide grouping of pixels for each magnificence mastermind (from dim to white), and keeping in mind that there are more noticeable pixels, the stature at the particular luminance degree is better.

\subsection{What is a color histogram?}

A color histogram of a photo addresses the spread of the bit of tints inside the photograph. It indicates one of a kind styles of shades showed up and the extent of pixels in each sort of the tints appeared. The association among a shading histogram and a luminance histogram is that a tinge histogram may be likewise conveyed as "Three Color Histograms", each 
one of which exhibits the sparkle dispersal of each man or woman Red/Green/Blue shading channel.

\subsection{Edge orientation histogram:}

The essential idea on this progression is to develop a histogram with the directions of the angles of the edges (outskirts or shapes). It is reasonable to recognize edges in a picture yet it on this we're interest inside the recognition of the edges. This is plausible trough Sobel administrators. The following 5 administrators should give a thought of the quality of the slope in five exact ways (Fig 4.1.).

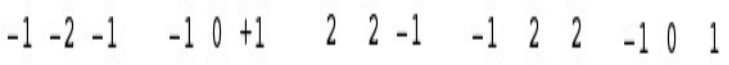

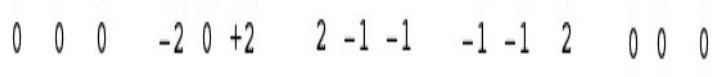

$$
\begin{aligned}
& +1+2+1 \quad-10+1 \quad-1-1-1 \quad-1-1-1 \quad 10-1 \\
& \text { (a) (b) (c) (d) } \\
& \text { (e) }
\end{aligned}
$$

content based picture recovery piece. Utilizing picture database we will get recovered pictures.

Content based absolutely picture recovery is extremely old fashioned approach. In this undertaking we laid out a methods of systems of picture recovery this are Low Level Image Features, Relevant Feedback (Human Interaction), Semantic-based picture recuperation, Extraction of Color, Texture Feature Extraction, Wavelet-Based CBIR, Web Based Image Retrieval.

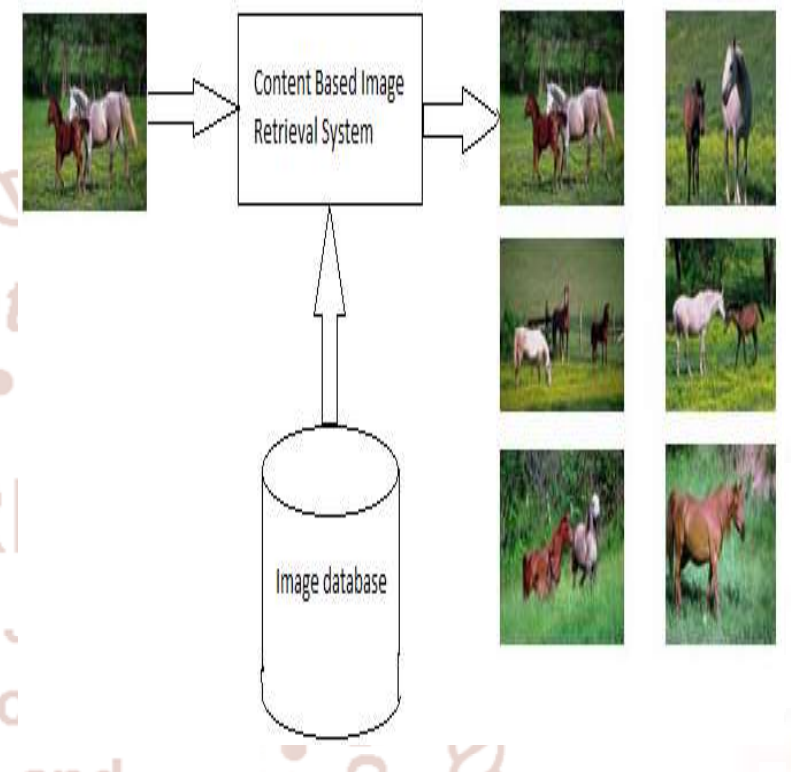

Fig. 1 Image Retrieval system by content

III.PROPOSED METHOD

\subsection{Feature Extraction of Minority Costume Image}

\section{A. Calculation of Color Histogram}

Color is a critical unmistakable property for every human conviction and pc creative and insightful and it's far broadly utilized as a part of picture recovery. The shading histogram is one of the greatest direct and the main shading trademark outline [21]. It has endowments of redesign invariant, pivot invariant and scale invariant and has been widely utilized as a part of picture recovery. In any case, it needs spatial realities. This undertaking incorporates spatial realities to it by utilizing joining the shading histograms for a few sub-pieces characterized in the minority attire picture. A proper shading zone and quantization should be point by point together with the histogram representation. In this venture, 3 hue spaces (RGB, HSV and CIE L*a*b*) with one of a kind evaluation go are utilized to check the execution of our Method. The exploratory outcomes in Tables 1three exhibit that the RGB shading region with $8 * 4 * 4=128$ measurement number is the tasteful 
decision in our system. For a photo with a size of $\mathrm{M} \times \mathrm{N}$, we set the shade evaluation wide assortment to $\mathrm{L}$ and mean the picture through the condition. The value assortment of $\mathrm{C} x \mathrm{y}(\mathrm{)}$, is [zero, ) $\mathrm{L}$. We separate the photo to $\mathrm{n}$ pieces. The shade estimations of each square is signified with the guide of $\mathrm{C} x \mathrm{y} I \mathrm{n}$ $\mathrm{I}(,),,([\mathrm{zero}),) \in$, then the hue histogram of each piece is depicted as:

$$
H_{c_{i}(x, y)}=\operatorname{num}_{j},(j=0,1, \ldots, L-1)
$$

Where numj is the number of pixels in a sub-block whose color value is quantified to $\mathrm{j}$.

\section{B. Calculation of Edge Orientation Histogram}

In the arrangement of idea on pc vision, edge recognition of photograph plays an essential capacity. These tasks amass a component descriptor to be specific edge introduction histogram, which can be obvious as a surface capacity and also a shape work. The exemplary territory location administrators are Sobel, Roberts, Prewitt and Canny. Sobel is one of the most extreme well known administrator [22], which is called after Irwin Sobel and Gary Feldman. The Sobel administrator depends on convolving the photo with a little, divisible, and whole number esteemed get out inside the flat and vertical rules and is therefore moderately less expensive as far as calculations. The administrator makes utilization of $3 \times 3$ portions which are convolved with the lauthentic photo to figure approximations of the subordinates - one for even, and one for vertical. In case we describe R, G, B as the unit vectors near to the R, G, B tomahawks in RGB shade district, the computations are according to the following: gxx , g yy and gxy are defined as dot products of the vectors mentioned above:

$$
\begin{gathered}
g_{x x}=g_{R x}^{2}+g_{G x}^{2}+g_{B x}^{2} \\
g_{y y}=g_{R y}^{2}+g_{G y}^{2}+g_{B y}^{2} \\
g_{x y}=g_{R x} \times g_{R y}+g_{G x} \times g_{G y}+g_{B x} \times g_{B y}
\end{gathered}
$$

Using the above notations, it can be seen that the maximum gradient orientation of point $(,) \mathrm{x} y$ is

$$
\varphi(x, y)=\frac{1}{2} \arctan \left(\frac{2 g_{x y}}{g_{x x}-g_{y y}}\right)
$$

And the gradient magnitude at $(\mathrm{x}, \mathrm{y})$ in the direction of $\phi($, , ) $\mathrm{y}$ given by

$$
\begin{aligned}
& G(x, y)=\left\{\frac { 1 } { 2 } \left[\left(g_{x x}+g_{y y}\right)\right.\right. \\
& +\left(g_{x x}-g_{y y}\right) \cos 2 \varphi \\
& \left.\left.+2 g_{x y} \sin 2 \varphi\right]\right\}^{1 / 2}
\end{aligned}
$$

Because $\tan () \tan () \alpha \alpha \pi= \pm$, if $\phi 0$ is a solution to Eq. (7), the $\phi 0 \pm \pi / 2$ will be a solution, too. Furthermore, G G $\phi \phi=+\pi$; therefore, G x y (, ) has to be computed only for values of $\phi$ in the half-open interval $[0,) \pi$. Because Eq. (7) provides two values $90^{\circ}$ apart, this equation associates a pair of orthogonal directions with each point $(,) \mathrm{x} y$ :

$$
\begin{aligned}
& G_{1}(x, y)=\left\{\frac { 1 } { 2 } \left[\left(g_{x x}+g_{y y}\right)\right.\right. \\
& +\left(g_{x x}-g_{y y}\right) \cos 2 \varphi \\
& \left.\left.+2 g_{x y} \sin 2 \varphi\right]\right\}^{1 / 2}
\end{aligned}
$$

$$
\begin{aligned}
& G_{2}(x, y)=\left\{\frac { 1 } { 2 } \left[\left(g_{x x}+g_{y y}\right)\right.\right. \\
& +\left(g_{x x}-g_{y y}\right) \cos 2\left(\varphi_{0}+\pi / 2\right) \\
& +2 g_{x y} \sin 2\left(\varphi_{0}\right. \\
& +\pi / 2)]\}^{1 / 2}
\end{aligned}
$$

$$
\begin{aligned}
g_{R y} & =\left[\begin{array}{ccc}
1 & 2 & 1 \\
0 & 0 & 0 \\
-1 & -2 & -1
\end{array}\right] \times R, g_{G y} \\
& =\left[\begin{array}{ccc}
1 & 2 & 1 \\
0 & 0 & 0 \\
-1 & -2 & -1
\end{array}\right] \times G, g_{B y} \\
& =\left[\begin{array}{ccc}
1 & 2 & 1 \\
0 & 0 & 0 \\
-1 & -2 & -1
\end{array}\right] \times B
\end{aligned}
$$

In practical applications, the maximum of the gradient direction is taken. Thus, we can denote the gradient direction $\phi(,,) \mathrm{x} y$ as follows:

$$
\begin{gathered}
\varphi(x, y) \\
= \begin{cases}\varphi_{0} & \text { ifmax }\left(G_{1}, G_{2}\right)=G_{2}(x, y) \\
\varphi_{0}+\pi / 2 & \text { if } \max \left(G_{1}, G_{2}\right)=G_{2}(x, y)\end{cases}
\end{gathered}
$$

To encourage usage, we wander it into the $\mathrm{c}$ program dialect period $[0,2] \pi$. After the edge introduction $\phi($, ) $\mathrm{x} y$ of each pixel has been figured, the introductions are consistently quantized into $\mathrm{m}$ pressing compartments, wherein $\mathrm{m}=12,18,24,30,36$. 
Information in Tables 1-three demonstrate that the 30 encloses utilized the RGB shade territory are additional appropriate for our system. Without a doubt, the introductions are quantized into 30 boxes, each like state of mind terms of $12^{\circ}$. We utilize histogram of part introduction to symbolize the element of minority dress photo. Since histogram is a measurable component, that is absence of the spatial part realities of the photo. Subsequently, on this task, sooner than the extraction of perspective introduction histogram trademark, we partition the minority dress photo with a size of $\mathrm{M} \times \mathrm{N}$ into $\mathrm{n}$ sub-pieces first. The region introduction estimations of each piece is indicated by means of $\phi \mathrm{i}(),,([0)$,$) x y I n \in$, then the limit introduction histogram of each square is depicted as

$$
H_{\varphi_{i}(x, y)}(j)=\operatorname{num}_{j},(j=0,1, \ldots, m-1)
$$

where numj is the number of pixels in a sub-block whose orientation value is quantified to $\mathrm{j}$.

\section{Comprehensive feature representation}

The far reaching highlight extraction calculation proposed in this undertaking can be spoken to as takes after: Step1: Divide the minority ensemble picture with a size of $M \times N$ into $n$ sub-hinders, the exploratory outcomes in Table 4 show that our strategy gets the best outcomes when $\mathrm{n}=2 \times 2$.

Step2: Calculate the color histogram of every subblock and then linearly combine them as $\mathrm{H}=[\mathrm{H}, \mathrm{H}$ $, \ldots, \mathrm{H}] \mathrm{C}$ x y C x y C x y Cx y $(),(),(),(,) ,12 \mathrm{n}$.

step3: Calculate the edge orientation histogram of each sub-block and then linearly combine them as follows:

$$
H_{\varphi(x, y)}=\left[H_{\varphi_{1}(x, y)}, H_{\varphi_{2}(x, y)}, \ldots . H_{\varphi_{n}(x, y)}\right]
$$

Step4: Linearly Combine all the histograms mentioned in step2 and step 3 as $\mathrm{H}=[\mathrm{H}, \mathrm{H}],([0),) \mathrm{C} \mathrm{x}$ y x y i i $(),(,) \phi$ in $\in$.

\subsection{Similarity Measurement}

The retrieval results can be again by using enlisting a closeness level of trademark vector among question picture and each photo inside the dataset.

This Project improve the Canberra remove as a fresh out of the plastic new separation metric by means of presenting a parameter $\lambda$, which make it more reasonable for our method.

$$
\begin{aligned}
& D(Q, T)=\lambda \sum_{i=1}^{C S A} \frac{\left|H_{i}(Q)\right|-H_{i}(T)}{1+H_{i}(Q)+H_{i}(T)} \\
&+(1 \\
&-\lambda) \sum_{j=1}^{C S B} \frac{\left|H_{i}(Q)\right|-H_{i}(T)}{1+H_{i}(Q)+H_{i}(T)}
\end{aligned}
$$

Where, $\mathrm{Hi}(\mathrm{Q})$ is the ith feature of query image $\mathrm{Q}$, $\mathrm{Hi}(\mathrm{T})$ is the $\mathrm{i}$ th feature of target image $\mathrm{T}$ in dataset.

\section{IV.EXECUTION RESULTS}

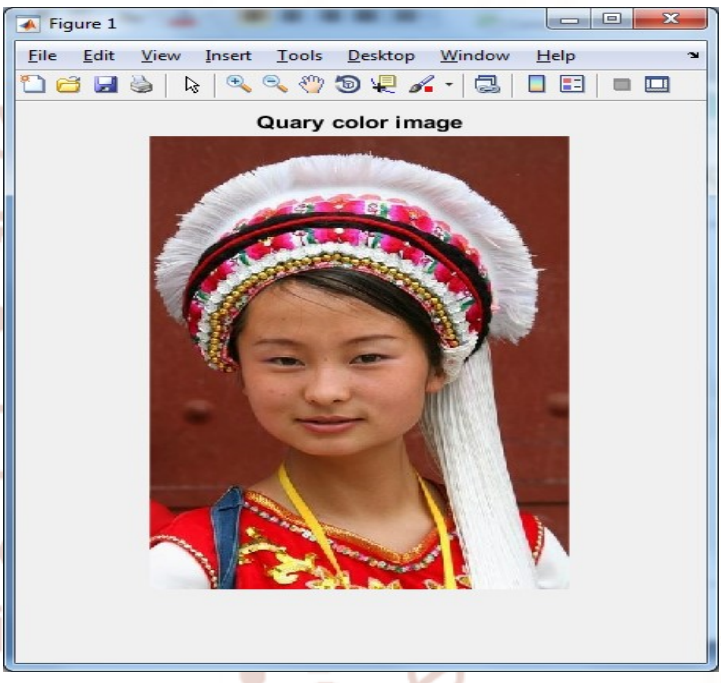

Fig.1 Query color image as input

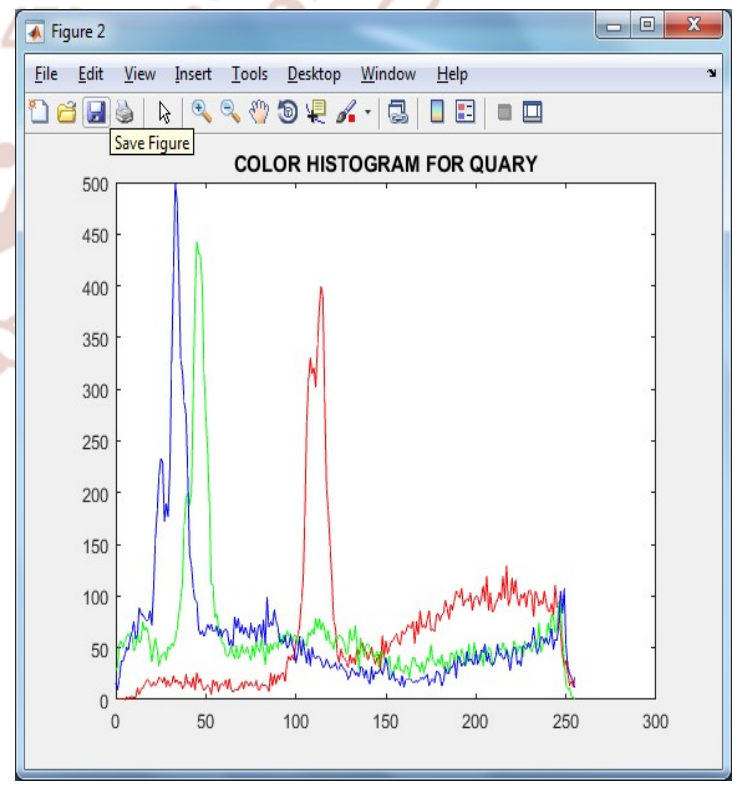

Fig.2 Color Histogram for Query image 


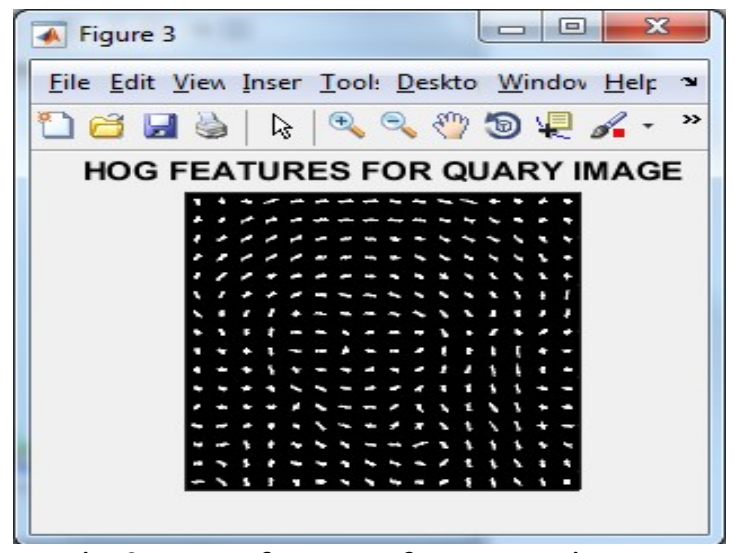

Fig.3 HOG features for Query image

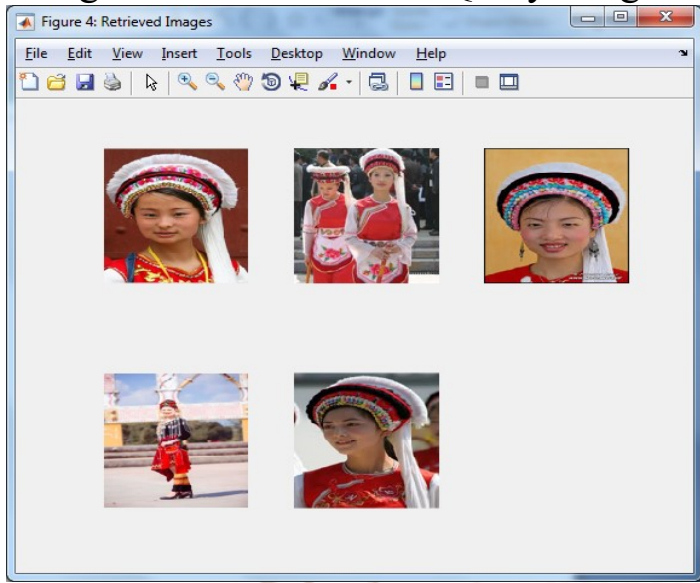

Fig.4 Retrieved Images for Query image

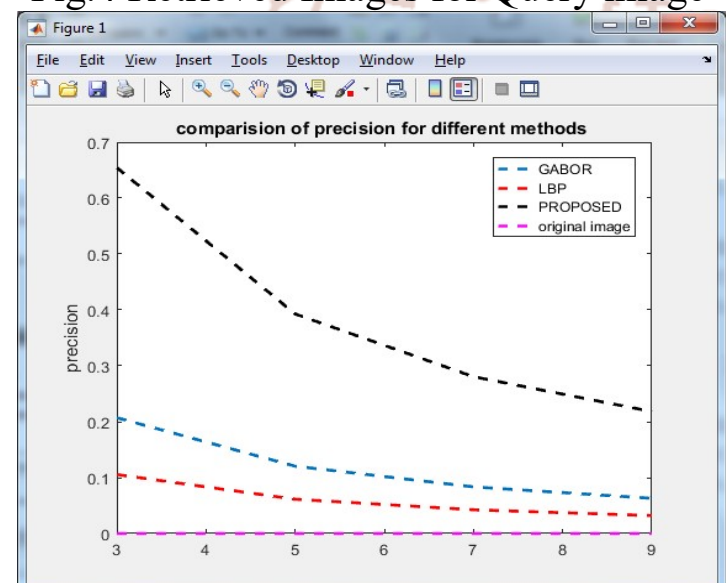

Fig.5 precision comparison of existing and proposed work

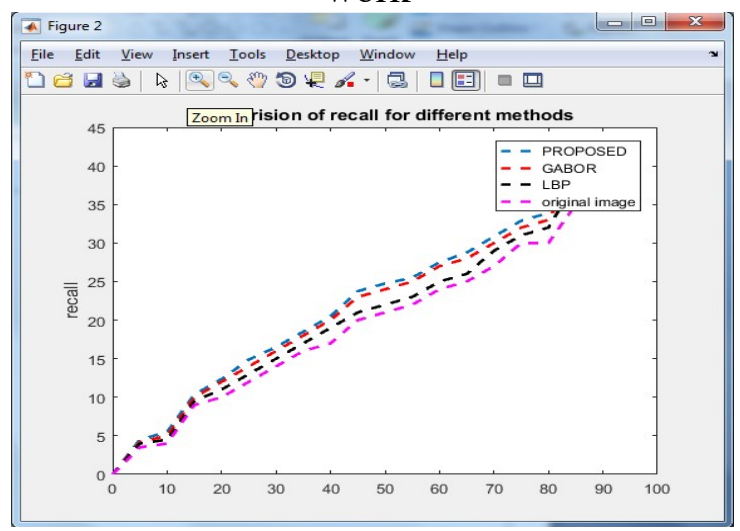

Fig.6 Recall comparison of existing and proposed work

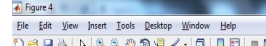

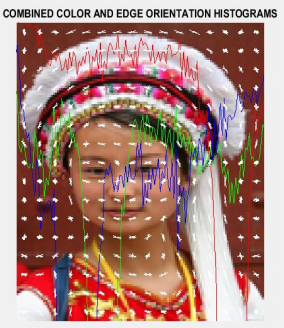

Fig. 7 Fusion of both color and Edge orientation histogram

\section{Advantages}

- Run time complexity is less.

- Objective parameters such as precison, recall are improved with the help of proposed work.

- As I used Canberra distance results are improved.

\section{Future Scope}

- In future we can use this work in general for CBIR and can prove the enhanced state of art of the proposed technique.

- Also, I can extend this technique for Video.

\section{V.CONCLUSION}

The purpose behind this execution is to acquire precise outcomes for minority outfit picture recovery by coordinating two procedures which utilizes all attributes, for example, shading, surface, shape and additionally spatial data. MATLAB execution result will demonstrate to us that our technique has great recovery execution and additionally solid flexibility. This coordinated method is vastly improved than existing state of workmanship procedures which are autonomously used for picture recovery, for example, GLCM, LBP, LDP, Gabor-based element descriptor, and invariant separation, $\mathrm{HOG}, \mathrm{MTH}, \mathrm{MSD}$ and $\mathrm{CDH}$ With the assistance of subjective and in addition target quality examination we can demonstrate that the proposed work outflanks in precision and is best condition of-craftsmanship system.

\section{REFERENCES}

1) M. J. Swain, D. H. Ballard, Color Indexing, International Journal of Computer Vision, Vol.7, No.1, 1991:11-32.

2) M. Stricker, and M. Orengo, "Similarity of color images", In SPIE Conference on Storage and Retrieval for Image and Video Databases, volume 2420, 1995, pp. 381-392, San Jose, USA. 
3) G. Pass, R. Zabin, J. Miller, Comparing Images Using Color Coherence Vectors, In ACM International Conference on Multimedia, Boston, MA, 1996: 65-73.

4) J. Huang,S. R. Kumar, M. Mitra ,W. J. Zhu ,R. Zabih, Image Indexing Using Color Correlograms, Conference on Computer Vision \& Pattern Recognition, 1997:762 - 768.

5) OAB Penatti, RD Silva Torres. Color descriptors for web image retrieval: a comparative study. The XXI Brazilian Symposium on Computer Graphics and Image Processing (SIBGRAPI '08), Campo Grande, Brazil, 12-15 Oct. 2008: 163-170.

6) R. Haralick, K. Shanmugam and I. Dinstein, Textural Features for Image Classification. IEEE Transactions on Systems, Man, and Cybernetics, VOL. SMC-3, No. 6, 1973(12).

7) T. Ojala, M. Pietikäinen, T. Mäenpää, Multiresolution Gray-Scale and Rotation Invariant Texture Classification with Local Binary Patterns, IEEE Transaction on Pattern Analysis and Machine Intelligence, 2002, 24(7):971-987.

8) T. Jabid, M. H. Kabir, O. Chae, Local Directional Pattern for Face Recognition, International Journal of Innovative Computing, Information and Control, Vol.8, No.4, April 2012.

9) Tai Sing Lee, Image Representation using 2D Gabor Wavelets, IEEE Transactions on pattern analysis and machine intelligence, VOL. 18, NO. 10, OCTOBER 1996.

10) M. K. Hu, Visual pattern recognition by moment invariant, IEEE Transactions on Information Theory, 1962, 8(2):179-187.

11) Eric Persoon, King-Sun Fu, Shape Discrimination Using Fourier Descrptors, IEEE Transactions on pattern analysis and machine intelligence, VOL. PAMI-8. NO. 3, MAY 1986.

12) Navneet Dalal, Bill Triggs, Histograms of Oriented Gradients for Human Detection, Proceedings of IEEE Computer Society Conference On Computer Vision and Pattern Recognition IEEE Press, 2005: 886- 893.

13) Guang-Hai Liu, Lei Zhang, Ying-Kun Hou, ZuoYong Li, JingYuYang, Image retrieval based on multi-texton histogram, Pattern Recognition, 44(2011):2123-2133.
14) Guang-Hai Liu, Zuo-Yong li, Lei Zhang, Yong $\mathrm{Xu}$, Image retrieval based on micro-structure descriptor, Pattern Recognition, 43(2010):23802389 .

15) Guang-Hai Liu, Jing-YuYang, Content-based image retrieval using color difference histogram, Pattern Recognition, 46 (2013):188-198.

16) J. Z. Wang, J. Li, G. Wiederholdy, simplicity: Semantics-sensitive Integrated Matching for Picture Libraries, IEEE Trans. on Pattern Analysis and Machine Intelligence, vol.23, no.9, pp.947963, 2001.

17) Choi Yoo-Joo, Kim Ku-Jin, Nam Yunyoung, Retrieval of Identical Clothing Images based on Local Color Histograms, 3rd International Conference on Convergence and Hybrid Information Technology, 2008, 1(1):818-823.

18) Ju-xiang Zhou, Xiao-dong Liu, Tian-wei Xu, Jianhou gan and Wanquan Liu. A new fusion approach for content based image retrieval with color histogram and local directional pattern, Pattern Recognition Letters.

19) I. Sobel, G. Feldman, "A $3 \times 3$ Isotropic Gradient Operator for Image Processing", presented at the Stanford Artificial Intelligence Project (SAIL) in 1968.

20) G. N. Lance, W. T. Williams, Mixed-data classificatory programs I, Agglomerative Systems, Australian Computer Journal, (1967): 15-20.

\section{Authors profile:}

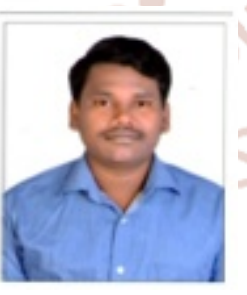

S SHIVA KUMAR Received his Bachelor's degree in 2015 in Electronics and Communication Engineering from CMR Institute of technology, India which is affiliated with JNTU Hyderabad, India. His areas of interest include Embedded System. He is pursuing his M.Tech in EMBEDDED SYSTEM from CMR Technical Campus.

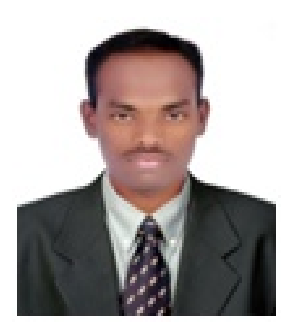

S MALLESH working as Assistant Professor in ECE department of CMRTC. He has an experience of 10 years In Teaching And administrative Fields in various Reputed Engineering colleges as Assistant professor. 